\title{
Live imaging of delamination in Drosophila shows that epithelial cell motility and invasiveness are independently regulated
}

\author{
Mikiko Inaki* and Kenji Matsuno
}

Department of Biological Sciences, Graduate School of Science, Osaka University, 1-1 Machikaneyama-cho, Toyonaka, Osaka 560-0043, Japan

*Corresponding author: minaki@bio.sci.osaka-u.ac.jp

\begin{abstract}
Delamination requires cells to undergo changes in cell-cell adhesion and in cell polarity, motility, and protrusions. This complex process must be precisely regulated during development as well as in pathogenic conditions. To determine the requirements for epithelial delamination, we analyzed the delamination of Drosophila ovary border cells, in which cells delaminate from the epithelial layer and begin to migrate collectively as is also seen in cancer metastasis. We used live imaging to examine cellular dynamics in delamination-defective mutants during the period in which delamination occurs in the wild-type ovary. We found that border cells in slow border cells (slbo), a delaminationdefective mutant, lacked the properties of invasive cellular extensions but acquired motility while JAK/STAT-inhibited border cells lost both cellular properties, suggesting that the invasiveness and motility required for delamination are regulated independently. Our reconstruction experiments showed that motility is not a prerequisite for acquiring invasiveness.
\end{abstract}

Key words: delamination, cell migration, locomotion, invasion 


\section{Introduction}

Delamination describes an event in which epithelial cells that normally form a layered structure become motile and detach from the organized cell layer. Epithelial cells acquire motility at appropriate times and places in the developing embryo and move where they are needed. Disrupting this precise control results in morphological abnormalities, cancer metastasis, and other pathological conditions (Stuelten et al., 2018; Thiery et al., 2009). During gastrulation, for example, the endodermal layer invaginates and epithelial cells detach from the layer and convert into mesenchymal cells to form the mesoderm (Ko and Martin, 2020; Thiery et al., 2009). In vertebrates, neural crest cells detach from the dorsal neural tube and migrate to form bone, neurons, glia, and other mesodermal cells (Gouignard et al., 2018; Szabó and Mayor, 2018). Delamination also occurs during cancer metastasis, in which grown tumor cells lose adhesion, digest epithelial-tissue basal membranes, and invade the body cavity (Stuelten et al., 2018; Thiery et al., 2009).

Delamination is also called epithelial-to-mesenchymal transition (EMT), reflecting the dynamic cellular changes in properties such as polarity, cell-cell adhesion, and cytoskeletal and membrane structures. However, the delaminated cells often retain epithelial properties such as cell polarity and adhesion, and the requirements for delamination remain elusive. To define the requirements for epithelial delamination, we analyzed the delamination of Drosophila border cells. Border cells are derived from the follicle-cell layer that surrounds germline cells, that is, the oocyte and supporting nurse cells in the developing egg chambers of the Drosophila ovary (Montell, 2006). The cells at the ends of the follicular layer, called pole cells, have distinct properties. The anterior pole cells secrete signals that activate JAK/STAT signaling in neighboring follicle cells (Silver and Montell, 2001). The follicle cells that receive JAK/STAT signaling start expressing slow border cells (slbo), which encodes a C/EBP transcription factor, and become motile border cells (Montell et al., 1992). After delaminating from the follicular layer, border cells migrate collectively towards the oocyte where they are essential for fertilization. Although their collective migratory process has been studied extensively as a model for cancer metastasis (Bianco et al., 2007; Cai et al., 2014; Cliffe et al., 2017; Dai et al., 2020; Inaki et al., 2012; Wang et al., 2010), little is known about the process by which border cells delaminate. 
Since cells undergo highly dynamic changes during delamination, we analyzed the process using live imaging. Delamination has been studied by live imaging in several systems, including EMT during neural crest cell development or at gastrulation (Clay and Halloran, 2013; Liu et al., 2017; Ramkumar et al., 2016; Saykali et al., 2019), and studies describe various morphological features and the involvement of actomyosin contractility. However, the hierarchy of gene regulation has not been analyzed. Here, we analyzed border-cell delamination at single-cell resolution through a live imaging system that allowed us genetically dissect and reconstruct the complex cellular processes involved in delamination. We found that epithelial cells acquired motility and invasiveness separately for delamination and that motility was not a prerequisite for invasiveness.

\section{Results and discussion}

\section{Locomotion is impaired upon JAK/STAT inhibition, but not in slbo mutants}

Delaminating border cells in the Drosophila ovary show dynamic cellular changes (Figure 1A). After the cells are specified and distinguished from other follicle cells, they start to send out small extensions (Figure 1A). Their affinity to other follicle cells changes and the shape of the cluster becomes more rounded (Figure 1A2-6). The cluster sends out front extensions, mostly single, in the direction the cluster will migrate (Figure 1A3-6). The extensions progressively elongate and contract, eventually forming a large protrusion, and the body of the cluster moves toward the protrusion and finally detaches from the epithelial layer (Figure 2A).

We investigated the cellular properties required for epithelial cell delamination using live imaging of border cells in delamination-defective mutants. To compare behavior in wild-type and delamination-defective cells during the period in which wildtype border cells become migratory, it is necessary to define developmental egg-chamber stages. Border cells are thought to delaminate around egg-chamber stage 8 to early stage 9 , but there are no objective markers for these stages. To obtain internal markers for eggchamber development, we measured several parameters, including the length and width of the oocyte and egg chamber, from movies of delamination in wild type. Of these parameters, we chose oocyte length as a suitable marker for egg-chamber stages because 
it consistently increases over time and appears to be a major contributor to egg-chamber growth (Figure 1B1-3). We analyzed 19 movies of wild-type delamination and defined the delamination time as the point when the entire cluster, including trailing cells, has detached from the follicular layer. The delamination time point varied in movies of wildtype cells. Since $85 \%$ of delamination events occurred when the oocyte was 50-70 microns long (Figure 1C), we defined an oocyte length of 50-70 microns as a marker for the wild-type delamination period (Figure 1C). We analyzed wild-type delamination movies for $30 \mathrm{~min}$ before the delamination point and movies of delamination-defective mutants for $30 \mathrm{~min}$ starting with the first frame in the wild-type delamination period. During this period, wild-type border cells form a round cluster that jiggles and forms an extension in the direction of migration (Figure 2A1-3, Figure 2-video 1). In delaminationdefective mutants, we first analyzed movies of egg chambers with JAK/STAT inhibition, which causes border cell differentiation to fail (Silver and Montell, 2001). Follicle cells neighboring the anterior pole cells use a receptor named Domeless (Dome) to receive ligands from the anterior pole cells and activate JAK/STAT signaling. To inhibit JAK/STAT signaling, a dominant-negative (DN) form of Dome receptor lacking the cytoplasmic domain (Dome ${ }^{\triangle \mathrm{CYT}}$ ) was expressed specifically in follicle cells, where border cells are differentiated, by using slbo-gal4 along with UAS-10×GFP as a cellular marker (Poukkula et al., 2011; Rørth et al., 1998; Silver and Montell, 2001). The JAK/STATinhibited border cells appeared to retain an epithelial morphology with apico-basal polarity, as do other follicle cells, and were not motile (Figure 2B1-3, Figure 2-video 2). Next, we examined border cells in a mutant for slbo, a downstream target of JAK/STAT signaling in border cells (Silver and Montell, 2001). We found that border cells in homozygotes for a hypomorphic allele, $s l b o^{1310}$, in which border-cell migration is almost completely abolished (Montell et al., 1992; Rørth et al., 1998), did not delaminate during the wild-type delamination period (Figure 2C). However, they behaved differently from border cells with JAK/STAT inhibition (Figure 2B,C), in that they formed a round cluster in which individual border cells moved back and forth in the cluster, similar to locomotion (Figure 2C1-3, Figure 2-video 3). We quantified this movement as angular velocity by measuring the movement of border cells relative to the center of the cluster (Figure 2F). The angular velocity was similar in slbo-mutant and wild-type border cells, but was lower 
in border cells with JAK/STAT inhibition - in fact, comparable with that of non-motile follicle cells in the posterior end (Figure 2D,G). We confirmed the cell motility in another allelic combination of $s l b o, s l b o^{1310} / s l b o^{e 7 b}$, in which border-cell migration is completely blocked (Figure2-figure supplement 1, Montell et al., 1992). These results indicate that slbo-mutant border cells acquired motility, allowing them to move about, even though they could not invade the egg chamber. To see whether this locomotion in individual cells was an active process involving cellular forces, we blocked actin polymerization with the actin polymerization inhibitor CytochalasinD. CytochalasinD treatment completely blocked border-cell delamination $(\mathrm{n}=13)$ and cell movement (Figure 2E,G, Figure 2-video 4), indicating that the observed locomotive behavior is an active process and is required for delamination.

\section{Invasiveness is impaired in slbo mutants}

Although the locomotive activity of slbo-mutant border cells is similar to that of wildtype, slbo-mutant border-cell clusters could not delaminate. They also had an evident defect - a lack of front extensions (Figure 3A,B). Wild-type border-cell clusters send extensions toward the direction of migration (Figure 3A). The protrusion extends and retracts over time, and although a cell with extensions is sometimes replaced by another, a cell with extensions is almost always present during the delamination period (Figure 2video 1). We quantified the front extensions using a previously described method (Poukkula et al., 2011) with slight modifications. Briefly, we binarized Z-stack images, separated the cluster body and extension by scanning a circle having a diameter slightly larger than that of the cell nucleus, and measured the size (length) and persistence (duration) of the front extensions. With this method, we found front extensions on wildtype border-cell clusters that were 18 microns long at most and persisted close to $30 \mathrm{~min}$ (analysis was done for $30 \mathrm{~min}$ ), but we found no front extensions on slbo-mutant border cells (Figure 3B,E,G,H, Figure 2-video 3). We concluded that slbo-mutant border cells acquire locomotion but not invasiveness, which might explain why these mutant bordercell clusters could not delaminate. Inhibiting actin polymerization by Cytochalasin D completely blocked the formation $(n=9)$ and maintenance $(n=17)$ of front extensions in wild-type border cells, showing that the formation and maintenance of front extensions 
are active processes of the actin cytoskeleton. These results indicate that for delamination, wild-type border cells acquire motility and invasiveness, both of which involve the actin cytoskeleton, and that invasiveness is regulated by slbo while motility is regulated by another factor(s) downstream of JAK/STAT signaling.

\section{Invasiveness induced by slbo is mediated partly by shg}

Slbo upregulates gene expression of shotgun (shg), which encodes E-cadherin (E-cad) in border cells (Mathieu et al., 2007; Tepass et al., 1996). We investigated a shg mutant, $s h g^{P B 4354}$, in which the upregulation in border cells is specifically suppressed (Mathieu et al., 2007). During the wild-type delamination period, shg $^{P B 4354}$-mutant border cells could not delaminate but showed normal locomotion, as did slbo-mutant border cells (Figure 3C, Figure3-figure supplement 1, Figure 3-video 1). Unlike slbo-mutant border-cell clusters, though, shg ${ }^{P B 4354}$-mutant border-cell clusters had front extensions (Figure 3C, Figure 3-video 1); however, the extensions were much shorter than wild-type in both length and duration (Figure 3E-H), suggesting that shg is required to form and maintain invasive extensions. These results explain why $s h g^{P B 4354}$-mutant border cells cannot delaminate during the wild-type delamination period. Next, we tested whether the overexpression of E-cad in border cells, which can rescue $\operatorname{shg}^{P B 4354}$ mutants, could rescue slbo-mutant phenotypes (Mathieu et al., 2007). The slbo-mutant border cells with forced E-cad expression had front extensions, suggesting that E-cad expression induced by Slbo is responsible for border-cell invasiveness (Figure 3D, Figure 3-video 1). However, the front extensions were still significantly shorter than in wild-type border cells (Figure $3 \mathrm{G}, \mathrm{H})$, and E-cad expression could not rescue the delamination impairment phenotype in slbo mutants, suggesting that other downstream components of slbo are involved in delamination.

\section{Invasive protrusion is required for reaching a distance}

We further investigated the role of front extensions in delamination by examining delamination in guidance-deficient mutants, since the extensions must be guided in the direction the delaminating border cells should migrate. Border-cell migration is guided by signaling through two receptor tyrosine kinases, PVR and EGFR, and blocking both 
receptors severely delays delamination (Duchek et al., 2001). To produce a guidancedeficient condition, DN forms of PVR and EGFR were specifically expressed in border cells. We examined guidance-deficient border cells during the wild-type delamination period and found that their front extensions were shorter and less persistent than wildtype (Figure 4A,D,E, Figure 4-video 1). The guidance-deficient border cells had normal locomotive behavior (Figure 4C). They eventually moved sideways and delaminated, though they took a less straightforward route than wild-type cells in most cases (Figure 4B, Figure 4-video 2). The cell movement and front extensions observed during the 30 min before the delamination point were similar to those in earlier stages (Figure 4C-E). The distance from the anterior tip of the egg chamber to the tip of the border-cell extension (defined as reach) was very similar in wild-type and guidance-deficient mutants at the point of delamination (Figure 4F). Both wild-type and guidance-deficient border-cell clusters reached about the same point before detaching completely, suggesting that the extension tips may have reached substrates they could grab hold of to aid in detaching the cluster and that it is the cluster's ability to reach such a substance rather than the actual length of the front extension that is important for delamination. Consistent with this idea, the maximum length of the front extension does not correlate with the point of delamination, and at the delamination point, the length of the front extension varies more than the distance from the tip of the extension to the anterior tip of the egg chamber (Figure 4-figure supplement 1). Although locomotion and front extensions were similar in shg-mutant and guidance-deficient border cells, shg-mutant border cells could not delaminate in the same time frame as guidance-deficient mutants. This might be due to less functional extensions that cannot grab hold of substrates on nurse cells. Dai et al. recently revealed that the border cell's preferential path is a multi-nurse-cell juncture (Dai et al., 2020). We found a hot spot close to the first multi-cell juncture with more than 3 nurse cells, suggesting that the preference for multi-cell junctures comes from not only more space but also more membrane to grab.

\section{Motility is not a prerequisite for invasiveness}

E-cad can induce invasive extensions in slbo-mutant border cells, which normally have no detectable extensions. We examined whether E-cad could induce invasive extensions 
in JAK/STAT-inhibited border cells, which lack locomotion. In normal conditions, border cells appear to send out extensions after acquiring motility (Figure 1A). To our surprise, JAK/STAT-inhibited follicle-like cells with forced E-cad expression sent out front extensions without changing the morphology of other follicle cells in most cases (Figure 5A, Figure 5-video 1). The length and duration of these front extensions were very like wild-type extensions (Figure 5D,E). These results indicated that cells can send out extensions without acquiring motility, again showing that motility and invasiveness are regulated independently. We further investigated this possibility by overexpressing Slbo in JAK/STAT-inhibited border cells. The JAK/STAT-inhibited follicle-like cells with forced Slbo expression produced front extensions, as did those with E-cad (Figure 5B, Figure 5-video 2). However, other border cells did not change shape and had less motility than wild-type (Figure 5C), supporting the idea that invasive extension does not require cell motility. These results also ruled out the possibility that residual Slbo activity conferred motility to the slbo ${ }^{1310}$ mutant. In the case of both E-cad and Slbo overexpression, induced invasiveness was not sufficient for delamination. Although both E-cad and Slbo overexpression can induce extensions, they have different effects. Extensions induced by E-cad were very thin and grew without ever retracting, suggesting that they lack machineries to produce traction forces (Figure 5A4). In contrast, Slbo induced extensions were thicker and retractable, suggesting that other downstream components of Slbo serve to make the extensions functional; this is consistent with the results of rescue experiments of slbo mutants (Figure 5B4,3D). In conclusion, these data revealed that motility and invasiveness are regulated independently and are required for delamination, and that motility is not a prerequisite for invasiveness.

\section{Materials and methods}

Fly strains

All flies were raised at $25{ }^{\circ} \mathrm{C}$ with standard media. We used the following fly strains: slbo-gal4 (Rørth et al., 1998), slbo ${ }^{1310}$ (Montell et al., 1992), slbo ${ }^{e 7 b}$ (null, Rørth, 1994), shg $^{P B 4354}$ (Mathieu et al., 2007), UAS-Dome $e^{\Delta C Y T}$ (Silver and Montell, 2001), UAS-shg (Mathieu et al., 2007), UAS-slbo (Rørth et al., 2000), UAS-DN-PVR, UAS-DN-EGFR (Duchek and Rørth, 2001), and UAS-10×GFP (Poukkula et al., 2011). We used the 
following genotypes for reconstruction experiments: $s l b o^{1310} /$ slbo $^{1310}$; UAS-shg / slbogal4, UAS-10×GFP, UAS-shg, UAS-Dome ${ }^{\triangle C Y T} /$ slbo-gal4, UAS-10×GFP, and UAS-slbo, UAS-Dome ${ }^{\triangle C Y T} /$ slbo-gal4, UAS-10 $\times$ GFP.

Live imaging and drug treatment

Live imaging was performed as previously described (Bianco et al., 2007). Images were acquired by confocal microscopy (SP5, Leica) with a $63 \times$ objective; sections were 2.5 $\mu \mathrm{m}$ apart and covered the cluster every $2 \mathrm{~min}$ for $2 \mathrm{~h}$. To inhibit actin polymerization, CytochalasinD (Sigma) was added into culture media at a final concentration of $25 \mu \mathrm{M}$.

Image analysis and quantification

For egg-chamber staging, we used transmission images of single $\mathrm{Z}$ slices showing the maximum egg chamber area and measured the width and height of the egg chamber and the oocyte. We defined an oocyte width of 50-70 microns as a marker for the wild-type delamination period. We analyzed movies for $30 \mathrm{~min}$ before the delamination point for wild-type Drosophila and for mutants, the first $30 \mathrm{~min}$ beginning with the first frame in the wild-type delamination period. For guidance-receptor mutants, we analyzed both time periods. To analyze single-cell movement, we tracked individual cells using nuclear position, marked by absence of GFP expression. We tracked the center of anterior pole cells as the cluster center. We measured border cells' change in angle relative to the cluster center using an Image $\mathbf{J}$ macro, and calculated the angular velocity. We analyzed front extensions using customized macros slightly modified from Poukkula et al. 2011. After making binarized images, we deleted starched cells, attaching border-cell clusters manually, then performed extension analysis as described previously (Poukkula et al., 2011). We could not use the same method for rescue experiments for JAK/STAT inhibition because the border cells did not form a round cluster, so we separated extensions by drawing a line where the extension base touched other border cells and measured the length and duration of the extensions. Statistical analyses were performed using Welch's or Student's t tests depending on the results of verification of standard deviation by $\mathrm{F}$ test. We calculated two-sided $\mathrm{p}$ values. 


\section{Acknowledgements}

We would like to thank Pernille Rørth and the Institute of Molecular and Cell Biology in Singapore for their support. This work was supported by Sumitomo, Futaba, and Takeda Science Foundations.

\section{References}

Bianco, A., Poukkula, M., Cliffe, A., Mathieu, J., Luque, C.M., Fulga, T.A., and Rørth, P. (2007). Two distinct modes of guidance signalling during collective migration of border cells. Nature 448, 362-365. 10.1038/nature05965.

Cai, D., Chen, S.C., Prasad, M., He, L., Wang, X., Choesmel-Cadamuro, V., Sawyer, J.K., Danuser, G., and Montell, D.J. (2014). Mechanical feedback through E-cadherin promotes direction sensing during collective cell migration. Cell 157, 1146-1159. 10.1016/j.cell.2014.03.045.

Clay, M.R., and Halloran, M.C. (2013). Rho activation is apically restricted by Arhgap1 in neural crest cells and drives epithelial-to-mesenchymal transition. Development 140, 3198-3209. 10.1242/dev.095448.

Cliffe, A., Doupé, D.P., Sung, H., Lim, I.K.H., Ong, K.H., Cheng, L., and Yu, W. (2017). Quantitative 3D analysis of complex single border cell behaviors in coordinated collective cell migration. Nature Communications 8 .

Dai, W., Guo, X., Cao, Y., Mondo, J.A., Campanale, J.P., Montell, B.J., Burrous, H., Streichan, S., Gov, N., Rappel, W.J., and Montell, D.J. (2020). Tissue topography steers migrating. Science 370, 987-990. 10.1126/science.aaz4741.

Duchek, P., and Rørth, P. (2001). Guidance of cell migration by EGF receptor signaling during Drosophila oogenesis. Science 291, 131-133. 10.1126/science.291.5501.131.

Duchek, P., Somogyi, K., Jékely, G., Beccari, S., and Rørth, P. (2001). Guidance of cell migration by the Drosophila PDGF/VEGF receptor. Cell 107, 17-26. 10.1016/s0092-8674(01)00502-5.

Gouignard, N., Andrieu, C., and Theveneau, E. (2018). Neural crest delamination and migration: Looking forward to the next 150 years. Genesis 56, e23107. 10.1002/dvg.23107.

Inaki, M., Vishnu, S., Cliffe, A., and Rørth, P. (2012). Effective guidance of collective migration based on differences in cell states. Proc Natl Acad Sci U S A 109, 2027-2032. 10.1073/pnas.1115260109.

Ko, C.S., and Martin, A.C. (2020). The cellular and molecular mechanisms that establish the mechanics of Drosophila gastrulation. Curr Top Dev Biol 136, 141-165. 10.1016/bs.ctdb.2019.08.003.

Liu, J.A., Rao, Y., Cheung, M.P.L., Hui, M.N., Wu, M.H., Chan, L.K., Ng, I.O., Niu, B., Cheah, K.S.E., Sharma, R., et al. (2017). Asymmetric localization of DLC1 defines avian trunk neural crest 
polarity for directional delamination and migration. Nat Commun 8, 1185. 10.1038/s41467-01701107-0.

Mathieu, J., Sung, H.H., Pugieux, C., Soetaert, J., and Rorth, P. (2007). A sensitized PiggyBacbased screen for regulators of border cell migration in Drosophila. Genetics 176, 1579-1590. 10.1534/genetics.107.071282.

Montell, D.J. (2006). The social lives of migrating cells in Drosophila. Curr Opin Genet Dev 16, 374-383. 10.1016/j.gde.2006.06.010.

Montell, D.J., Rorth, P., and Spradling, A.C. (1992). slow border cells, a locus required for a developmentally regulated cell migration during oogenesis, encodes Drosophila C/EBP. Cell 71, 51-62. 10.1016/0092-8674(92)90265-e.

Poukkula, M., Cliffe, A., Changede, R., and Rørth, P. (2011). Cell behaviors regulated by guidance cues in collective migration of border cells. J Cell Biol 192, 513-524. 10.1083/jcb.201010003.

Ramkumar, N., Omelchenko, T., Silva-Gagliardi, N.F., McGlade, C.J., Wijnholds, J., and Anderson, K.V. (2016). Crumbs2 promotes cell ingression during the epithelial-to-mesenchymal transition at gastrulation. Nat Cell Biol 18, 1281-1291. 10.1038/ncb3442.

Rørth, P. (1994). Specification of C/EBP function during Drosophila development by the bZIP basic region. Science 266, 1878-1881.

Rørth, P., Szabo, K., Bailey, A., Laverty, T., Rehm, J., Rubin, G.M., Weigmann, K., Milán, M., Benes, V., Ansorge, W., and Cohen, S.M. (1998). Systematic gain-of-function genetics in Drosophila. Development 125, 1049-1057.

Rørth, P., Szabo, K., and Texido, G. (2000). The level of C/EBP protein is critical for cell migration during Drosophila oogenesis and is tightly controlled by regulated degradation. Mol Cell 6, 23-30. 10.1016/s1097-2765(05)00008-0.

Saykali, B., Mathiah, N., Nahaboo, W., Racu, M.L., Hammou, L., Defrance, M., and Migeotte, I. (2019). Distinct mesoderm migration phenotypes in extra-embryonic and embryonic regions of the early mouse embryo. Elife $8.10 .7554 /$ eLife.42434.

Silver, D.L., and Montell, D.J. (2001). Paracrine signaling through the JAK/STAT pathway activates invasive behavior of ovarian epithelial cells in Drosophila. Cell 107, 831-841. 10.1016/s0092-8674(01)00607-9.

Stuelten, C.H., Parent, C.A., and Montell, D.J. (2018). Cell motility in cancer invasion and metastasis: insights from simple model organisms. Nat Rev Cancer 18, 296-312. 10.1038/nrc.2018.15.

Szabó, A., and Mayor, R. (2018). Mechanisms of Neural Crest Migration. Annu Rev Genet 52, 4363. 10.1146/annurev-genet-120417-031559.

Tepass, U., Gruszynski-DeFeo, E., Haag, T.A., Omatyar, L., Török, T., and Hartenstein, V. (1996). shotgun encodes Drosophila E-cadherin and is preferentially required during cell rearrangement 
in the neurectoderm and other morphogenetically active epithelia. Genes Dev 10, 672-685. 10.1101/gad.10.6.672.

Thiery, J.P., Acloque, H., Huang, R.Y., and Nieto, M.A. (2009). Epithelial-mesenchymal transitions in development and disease. Cell 139, 871-890. 10.1016/j.cell.2009.11.007.

Wang, X., He, L., Wu, Y.I., Hahn, K.M., and Montell, D.J. (2010). Light-mediated activation reveals a key role for Rac in collective guidance of cell movement in vivo. Nat Cell Biol 12, 591597. $10.1038 / \mathrm{ncb} 2061$. 

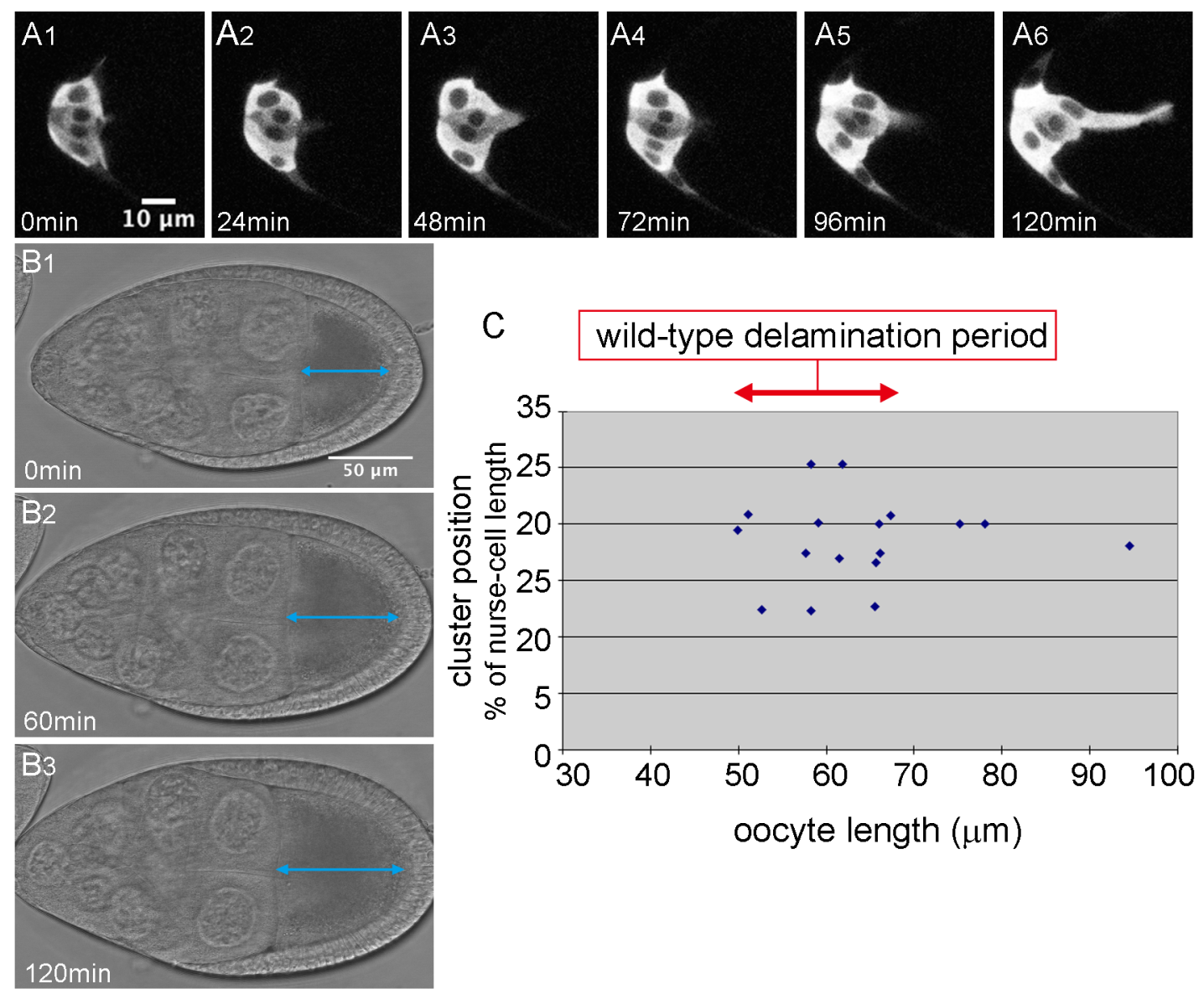

oocyte length $(\mu \mathrm{m})$

Figure 1.

Delamination is a complex cellular process. (A) A time series showing the delamination process in wild-type Drosophila border cells. (B) A time series of transmission images of the egg chamber during delamination. Blue arrows indicate oocyte length, which increased with time. (C) Oocyte length at the delamination point was determined from 17 videos of wild-type border cells. We defined an oocyte length of 50-70 $\mu \mathrm{m}$ as a marker of the wild-type delamination period. Elapsed time from the start of the video is shown at the bottom left. For all images, anterior is left. 


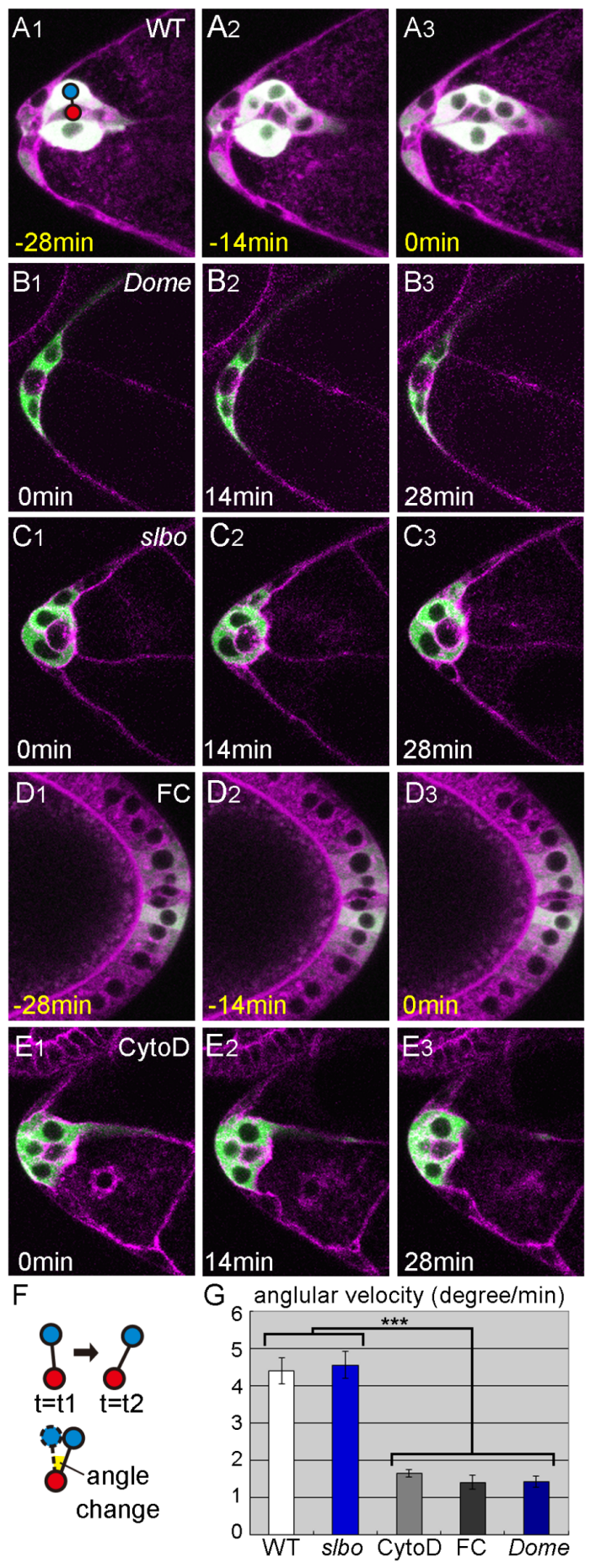

Figure 2.

Locomotion is impaired upon JAK/STAT inhibition in wild-type but not in slbo-mutant border cells. (A-E) Single Z slices from movies showing the delamination of wild-type (A), DN-Dome expressing (B), slbo-mutant (C), and CytochalasinD-treated (D) border cells and a movie of posterior follicle cells (E). Red and blue circles indicate the cluster 
center and a border cell nucleus, respectively. (F) A schematic showing how cell movement was quantified by measuring the change in the angle of the border cell nucleus (blue circle) relative to the cluster center (red circle). (G) The angular velocity of wildtype (WT), DN-Dome-expressing (Dome), slbo-mutant (slbo), and CytochalasinDtreated (CytoD) border cells relative to the cluster center, and the angular velocity of posterior follicle cells relative to the posterior pole cell center (FC). $12 \leqq$ number of cells (n) $\leqq 24,6 \leqq$ number of egg chambers $(\mathrm{N}) \leqq 12$. Error bars indicate SEM. ***, $\mathrm{p}<0.001$. Time shown at the lower left indicates elapsed time from the start of the video (white) and time prior to the delamination point (yellow). For all images, anterior is left. 


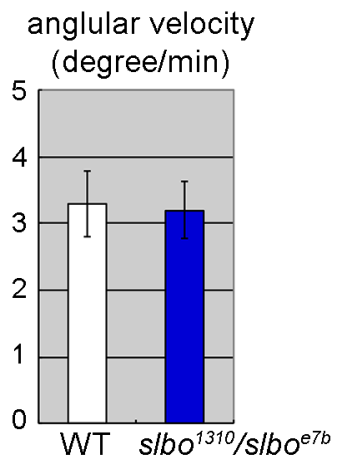

Figure 2-figure supplement 1

Angular velocity for the following border cells: wild-type (WT) and $s l b o^{1310} /$ slbo $^{e 7 b}$. Angular velocities were comparable in these conditions. $n=10, N=5$. Error bars indicate SEM. 

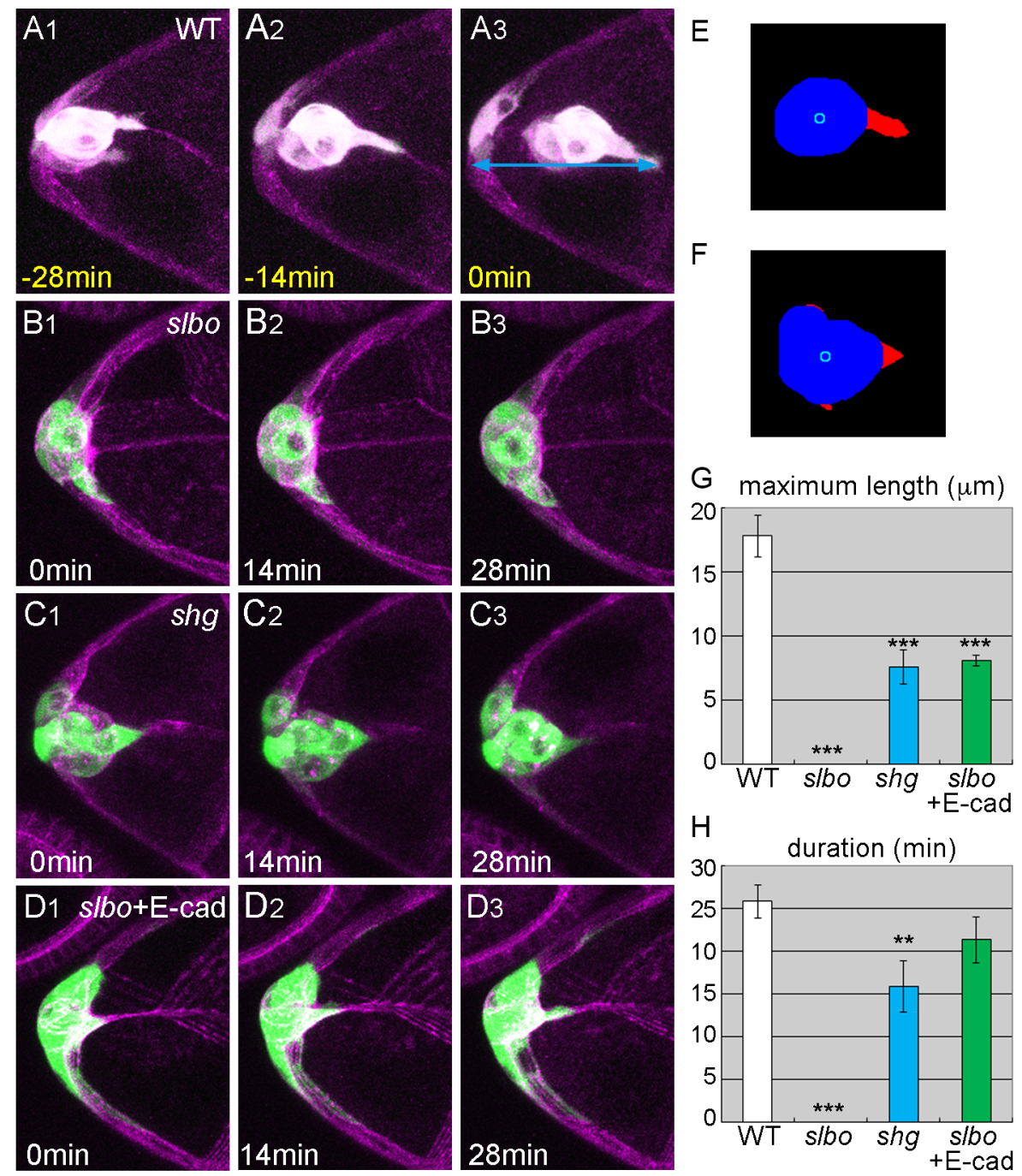

Figure 3

Invasiveness is regulated by slbo partly though shg. (A-D) Z stacks from delamination movies of border cells, as follows: wild-type (A), slbo-mutant (B), shg-mutant (C) and slbo-mutant with E-cad expression (D). Light blue arrow indicates the distance (reach) from the anterior end of the egg chamber to the tip of the border-cell extension at delamination (A3). (E,F) Cluster body (blue) and extensions (red) separated by image analysis from the images shown in (A2,C2). Light blue circles: the centroid of the cluster body. $(\mathrm{G}, \mathrm{H})$ The maximum length $(\mathrm{G})$ and duration $(\mathrm{H})$ of the front extensions of bordercell clusters, as follows: wild-type (WT), slbo-mutant (slbo), shg-mutant (shg), and slbomutant with E-cad expression (slbo+E-cad). $6 \leqq \mathrm{~N} \leqq 14$. Error bars indicate SEM. ***, $\mathrm{p}<0.001, * *, \mathrm{p}<0.01$. Time shown at the lower left indicates time elapsed from the start 
of the video (white) and the time prior to the delamination point (yellow). For all images, anterior is left. 


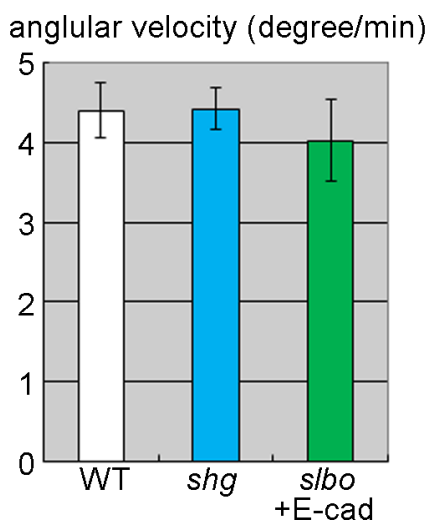

Figure 3-figure supplement 1

Angular velocity for the following border cells: wild-type (WT), shg-mutant (shg), and slbo-mutant with E-cad expression (slbo+E-cad). Angular velocities were comparable in these conditions. $10 \leqq \mathrm{n} \leqq 26,5 \leqq \mathrm{~N} \leqq 13$. Error bars indicate SEM. 

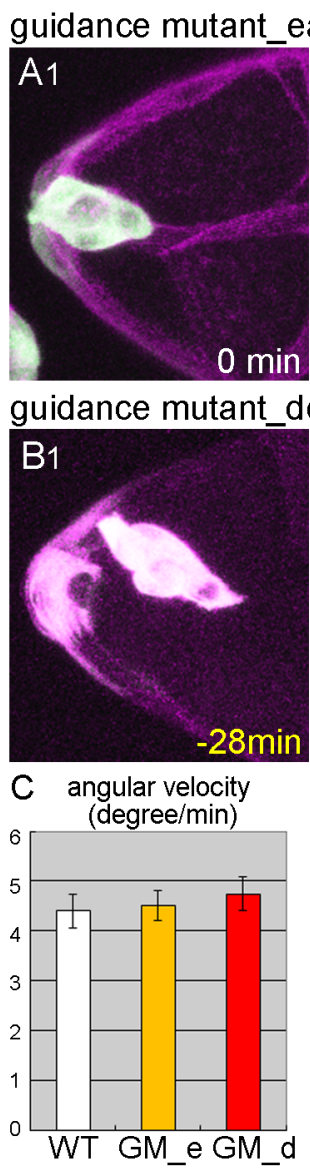

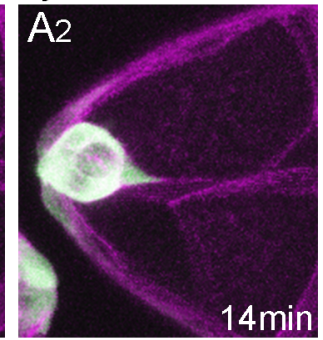

elaminating
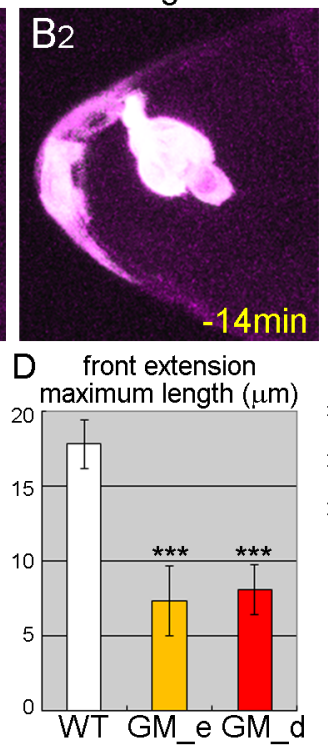
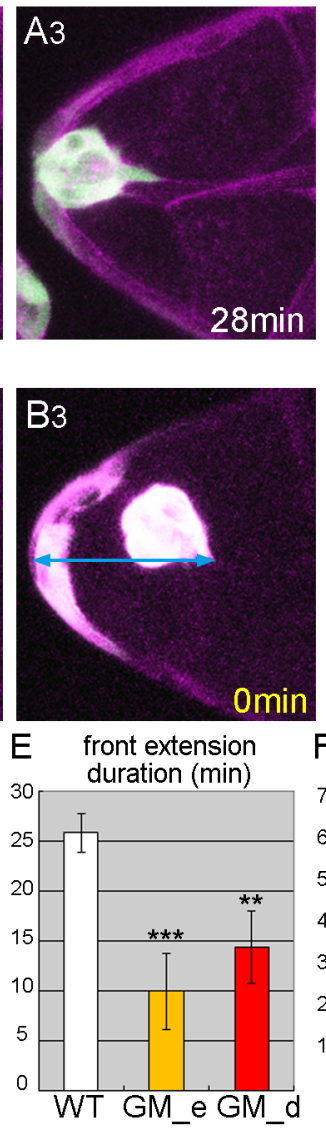

F cluster reach

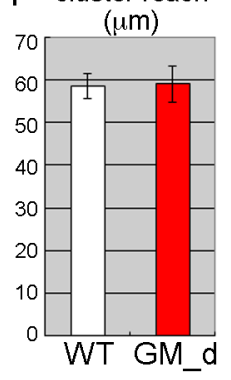

Figure 4

Invasive protrusion is required to reach a distance. (A,B) $\mathrm{Z}$ stacks of guidance-deficient mutant movies in the wild-type (A), and actual (B) delamination period. Light blue arrow indicates from the anterior end of the egg chamber to the reach of the border-cell cluster at delamination (B3). (C-E) Angular velocity (C), maximum length (D) and persistence (E) of front extensions of border-cell clusters in wild-type (WT), and guidance-deficient mutants in the wild-type (GM_e) and actual (GM_d) delamination periods. (C) $16 \leqq \mathrm{n} \leqq$ $24,8 \leqq \mathrm{~N} \leqq 12$. (D,E) $8 \leqq \mathrm{~N} \leqq 14$ (F) The distance from the anterior end of the egg chamber to the tip of the border-cell extension at the delamination point for wild-type (WT) and the guidance-deficient mutant (GM_d). $8 \leqq \mathrm{~N} \leqq 13$. Error bars indicate SEM. $* * *, \mathrm{p}<0.001, * *, \mathrm{p}<0.01$. Time shown at the lower left indicates time elapsed from the start of the video (white) and time prior to delamination (yellow). For all images, anterior is left. 

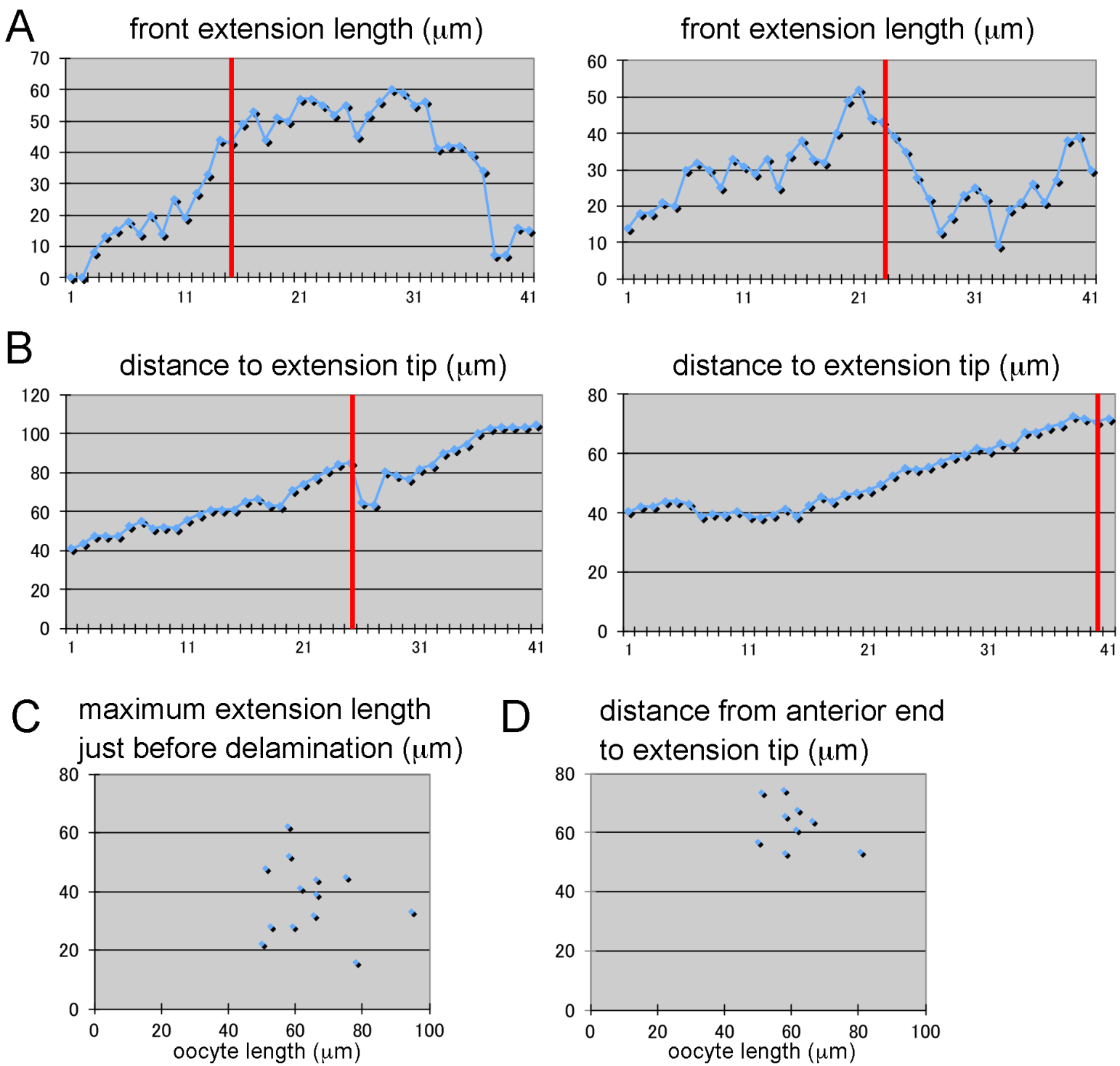

Figure 4-figure supplement 1

$(A, B)$ Examples of changes in front extension length (A) and in cluster reach (the distance between the anterior end of the egg chamber and the extension tip) (B) during delamination. $\mathrm{X}$ axis: time frame. Red lines: delamination point. Front extension length varied quite a bit, while cluster reach tended to increase over time. The maximum extension length did not correlate with the delamination point. (C,D) The maximum extension length (C) and the cluster reach (D) just before delamination. Length varied more than reach. 

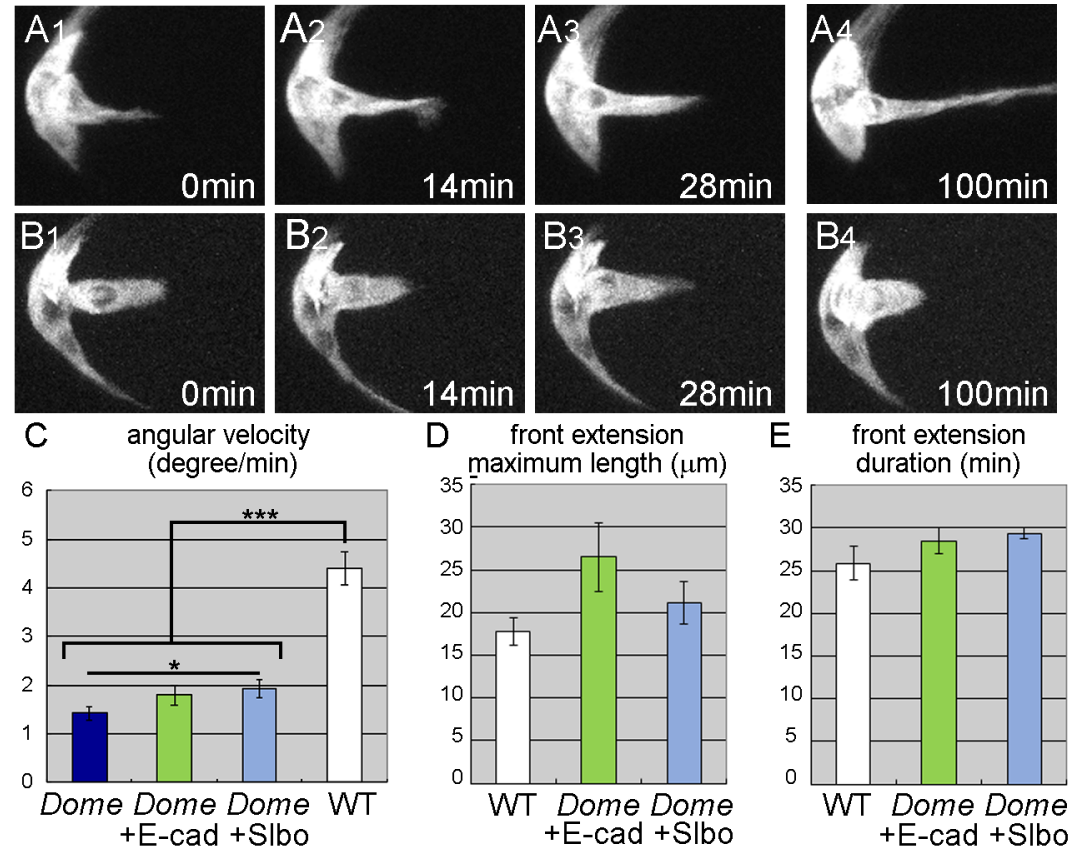

Figure 5

Motility is not a prerequisite for invasiveness. (A,B) $\mathrm{Z}$ stacks from movies of border cells expressing DN-Dome with E-cad (A) and Slbo (B). E-cad and Slbo induced front extensions in JAK/STAT-inhibited border cells. (C-E) Angular velocity and (C) the maximum length (D) and duration of front extensions in wild-type (WT) or DN-Dome expressing (Dome) border-cell clusters with either E-cad (Dome+E-cad) or Slbo (Dome + Slbo) expression. (C) $16 \leqq \mathrm{n} \leqq 24,8 \leqq \mathrm{~N} \leqq 12$. (D,E) $6 \leqq \mathrm{~N} \leqq 14$. Error bars indicate SEM. $* * *, \mathrm{p}<0.001, * *, \mathrm{p}<0.01$. Time elapsed from the start of the video is indicated at the bottom right. For all images, anterior is left. 


\section{Videos}

Figure 2-video 1

Delamination of wild-type border cells.

Figure 2-video 2

JAK/STAT inhibited border cells in the wild-type delamination period.

Figure 2-video 3

slbo-mutant border cells in the wild-type delamination period.

Figure 2-video 4

Cytochalasin D-treated border cells in the wild-type delamination period.

Figure 3-video 1

shg-mutant border cells in the wild-type delamination period.

Figure 3-video 2

slbo-mutant border cells with E-cad expression in the wild-type delamination period.

Figure 4-video 1

Guidance-deficient border cells in the wild-type delamination period.

Figure 4-video 2

Guidance-deficient border cells in the actual delamination period.

Figure 5-video 1

JAK/STAT inhibited border cells with E-cad expression in the wild-type delamination period.

Figure 5-video 2 
JAK/STAT inhibited border cells with Slbo expression in the wild-type delamination period. 